Original article

\title{
The effect of controlled reperfusion on experimental ovarian torsion
}

\author{
Cornelia Lazăr ${ }^{1}$, Marin Vozian ${ }^{1}$, Valeriana Pantea ${ }^{1}$, Inna Svet ${ }^{1}$, Anna Mishina ${ }^{2}$, Olga Tagadiuc ${ }^{1}$ \\ ${ }^{1}$ Nicolae Testemitanu State University of Medicine and Pharmacy, Chisinau, Republic of Moldova \\ ${ }^{2}$ Mother and Child Institute, Chisinau, Republic of Moldova
}

Received 28 March 2019, Revised 5 May 2019, Accepted 8 August 2019

(C) 2019, Lazăr C., Vozian M., Pantea V., Svet I., Mishina A., Tagadiuc O.

(C) 2019, Russian Open Medical Journal

Abstract: The objective of our research was to study the effect of controlled reperfusion on lactate and advanced oxidation protein products (AOPP) in experimental ovarian torsion (OT), and to evaluate the possibility to reduce the reperfusion injuries applying the ONOFF technique of reperfusion.

Material and Methods - Lactate and AOPP were measured in serum and ovarian homogenates of 70 females rats, divided equally into 7 groups: no intervention (nr. 1); laparotomy (nr. 2); 3 hours OT (nr. 3); 3 hours OT +1 hour (nr. 4) or 24 hours (nr. 6) of simple reperfusion (SR); 3 hours OT +1 hour (nr. 5) or 24 hours (nr. 7) of controlled reperfusion (ON-OFF). The results were analyzed using Welch's ANOVA.

Results - OT produced an increase of serum $(66 \%, p<0.001)$ and homogenate $(38 \%, p=0.006)$ lactate, that tended to return to baseline in 24 hours despite the type of reperfusion. AOPP had grown in OT animals serum $(20 \%, p=0.007)$ and homogenate $(26 \%, p>0.05)$ and continued to rise in the serum after SR, while in ON-OFF technique groups - decreased by about $20 \%$ ( $<<0.01$ ). Homogenate AOPP levels rised after reperfusion in all groups.

Conclusion - Lactate is a biomarker for ischemia in OT. Increased AOPP in experimental groups indicates that there is a degree of oxidative stress that can affect the cells after the restoration of the blood flow. Therefore, the correct management of the condition that reduces reactive oxygen species production, such as controlled method of reperfusion, could be appropriate to prevent additional injuries.

Keywords: ovarian torsion, controlled reperfusion, reperfusion injury, lactate, advanced oxidation protein products.

Cite as Lazăr C, Vozian M, Pantea V, Svet I, Mishina A, Tagadiuc O. The effect of controlled reperfusion on experimental ovarian torsion. Russian Open Medical Journal 2019; 8: e0404.

Correspondence to Cornelia Lazăr. Address: Chair of Biochemistry and Clinical Biochemistry, Nicolae Testemitanu State University of Medicine and Pharmacy, 165, Stefan cel Mare si Sfant ave, Chisinau, Republic of Moldova, MD-2004. Phone: +37322205503. E-mail: cornelia.lazar@usmf.md.

\section{Introduction}

One of the gynecological emergencies that may affect women at any age is the torsion of the ovary [1]. The condition is due to the rotation of the ovary or/and fallopian tube that impair the normal blood flow [2]. The prevalence of the ovarian torsion is from 2.7 to $3.0 \%$ [3] and the incidence is $4.9 / 100000$ in young women under 20 [4].

This disease is difficult to be diagnosed because of its nonspecific clinical presentation $[5,6]$ that mimics other disorders [7]. Nowadays available non-surgical tools for diagnosis are not very helpful. The accuracy of ultrasounds examination is not high because sometimes Doppler imaging detect a normal blood flow to the adnexa in ovarian torsion [8], and this delay the diagnosis, that may lead to the loss of the ovary or complications such as peritonitis and even death [9]. Laparoscopy is the "gold standard" in diagnosing the torsion of the ovary $[6,10]$.

Surgical removal of the ovary was the main option for a long time, nowadays conservative management with detorsion is considered the best option in young women. But the restoration of the blood flow may induce additional lesions due to oxidative stress and a high reactive oxygen species (ROS) production [11]. Thus, the delayed diagnosis is a problem, but also are the further oxidative injuries after surgical intervention.

Ischemia is a condition that changes the metabolic pathways of cells to anaerobic ones and lactate amounts increase [12].

Advanced oxidation protein products (AOPP) are derivatives of albumin, fibrinogen and lipoproteins that are modified oxidatively. AOPP are present in physiological conditions, in the blood of healthy persons, but in low concentration. An increased production of ROS changes the native structure of proteins transforming them into pathological particles named AOPP [13].

Oxidative stress increases after an ischemic period. During the reperfusion phase ROS can grow the ischemic lesions suggesting that the reperfusion method is essential to prevent additional injuries.

The AOPP and lactate levels were not investigated till now in the context of controlled reperfusion in ovarian torsion. The aim of our research was to study the effect of controlled reperfusion on 
lactate and AOPP levels in experimental ovarian torsion (OT) and to evaluate the capacity of the ON-OFF technique of reperfusion to reduce the reperfusion injuries.

\section{Material and Methods}

Animals

The experiment was performed in the Laboratory of Biochemistry of the Nicolae Testemitanu State University of Medicine and Pharmacy.

Our study involved 70 rats (Rattus albicans), females from the vivarium of the Nicolae Testemitanu State University of Medicine and Pharmacy.

The rats (body weight 180-265 grams) were randomly distributed into seven groups, each group including ten animals: 1 - no intervention (control); 2 - only laparotomy (sham); 3 - the ovaries were exposed to ischemia twisting the adnexa for 3 hours (OT group); 4 - OT for 3 hours, 1 hour simple reperfusion (SR); 5 OT for 3 hours, 1 hour controlled (ON-OFF) reperfusion (the first 120 seconds we opened and closed the clips on the ovarian pedicles in 10 seconds intervals, succeeded by SR); 6 - OT for 3 hours, 24 hours SR; 7 - OT for 3 hours, 24 hours ON-OFF reperfusion.

\section{Surgical procedures}

All surgical manipulations were done under sterile conditions. The rats received intraperitoneal anesthesia $50 \mathrm{mg} / \mathrm{kg}, 10 \%$ ketamine hydrochloride sol. (Calypsol ${ }^{\circledR}$, Gedeon Richter, Hungary).

The surgical intervention that lasted approximately 15 minutes was accomplished after the rats' skin was disinfected using lodine povidone $100 \mathrm{mg} / \mathrm{ml}$ (Betadine ${ }^{\circledR}$, Egis Pharmaceuticals PLC, Hungary) and shaved. When animals were motionless after anesthesia in a supine position, a $2-2.5 \mathrm{~cm}$ laparotomy in the lower abdomen was performed.

The rats from the control group were not subject to ovarian torsion. They were only anesthetized, laparotomy performed, after which blood samples from abdominal aorta and ovaries were collected, then they were euthanized.

The sham group also underwent no ovarian torsion. After anesthesia the rats were subject to laparotomy, the abdomen cavity was left open for 15 minutes, then was closed using 3/0 silk sutures. Re-laparotomy was done after 3 hours under anesthesia. Ovaries and blood samples were taken after which the rats were euthanized.

In animals of the experimental groups 3-7 the ovaries were subject to OT for 3 hours, by twisting the annexes in the clockwise direction 3 times and fixing them to the abdominal wall with $3 / 0$ silk suture and closing after that the abdomen using $3 / 0$ silk sutures. After the torsion period the animals were re-anesthetized and their abdominal wall opened. In rats that belong to third group (OT group) the ovaries and blood from aorta was collected and according to the study protocol the rats were euthanized. The animals that belong to groups 4-7 underwent different reperfusions methods after the torsion period, as follows: group 4 - detorsion of the ovaries with 1 hour of simple reperfusion (SR), group 6 - detorsion with 24 hours SR, group 5 - detorsion with 1 hour controlled reperfusion (ON-OFF), group 7 - detorsion with 24 hours ON-OFF. The ON-OFF procedure was performed the first 2 minutes after the torsion period followed by simple reperfusion.
Using the clips on ovarian pedicles, we controlled the restoration of the blood flow gradually opening and closing them in 10 seconds intervals. After the detorsion procedure the abdomen was closed with $3 / 0$ silk sutures. After the reperfusion phase the rats underwent anesthesia, the abdominal wall was opened, then ovaries and blood samples from abdominal aorta collected. After the procedure the rats were euthanized.

\section{Biochemical analysis}

The blood samples were placed into tubes allowing to clot for 30 minutes, then centrifuged 10 minutes at $1500 \mathrm{rpm}$. The serum was stored at $-40^{\circ} \mathrm{C}$ until analysis.

The ovarian tissue $(0.2 \mathrm{~g})$ was homogenized in ice with $2 \mathrm{ml}$ of phosphate buffer $0,1 \mathrm{M}(\mathrm{pH}=7.4)$, centrifuged at $4{ }^{\circ} \mathrm{C}, 3000 \mathrm{rpm}$ for 15 minutes. The supernatant stored at $-40^{\circ} \mathrm{C}$ until analysis.

Lactate amounts were determined using the spectrophotometric method according to the instructions of the kit of EliTech Group (France).

AOPP levels were measured spectrophotometrically using the Capeillère-Blandin C., et al. method [14].

\section{Statistical analysis}

SPSS 20.0 software was used to analyse the results. The mean and standard deviation were used as descriptive statistical methods. The individual group biochemical parameters were examined for normality using Shapiro-Wilk and KolmogorovSmirnov tests and were considered normally distributed ( $p>0.05)$. Levene's test was applied to study the homogeneity of variance. The data obtained in different groups were compaired using Welch's ANOVA. Games-Howell post hoc test was used. Spearman test was applied to identify the correlations between serum and homogenates biochemical markers. Statistically significant was considered the value of $p<0.05$.

\section{Results}

The results are presented as mean and standard deviation in Table 1 for ovarian homogenates and Table 2 for blood serum.

Table 1. Lactate and AOPP levels in ovarian homogenates

\begin{tabular}{|c|c|c|}
\hline Group & Lactate, $\mathrm{mmol} / \mathrm{g} . \mathrm{prot}$. & AOPP, $\mu \mathrm{mol} / \mathrm{g}$. prot. \\
\hline 1 - control & $2.893 \pm 0.283$ & $5.695 \pm 0.781$ \\
\hline 2 - sham & $2.918 \pm 0.240$ & $5.804 \pm 1.179$ \\
\hline 3 - ischemia (OT) & $4.002 \pm 0.667 * \mathrm{pDs}$ & $7.183 \pm 1.566 \beta$ ss J \\
\hline $4-1 h$ SR & $3.933 \pm 0.395 * *$ DDD SSS & $9.434 \pm 0.747 * * * \operatorname{pdp} \delta$ \\
\hline 5 - $1 \mathrm{~h}$ ON-OFF & $3.907 \pm 0.147 * * *$ dod sss J & $8.401 \pm 1.288 * * \mathrm{pD}$ \\
\hline $6-24 h$ SR & $2.957 \pm 0.130 \delta \beta \beta \beta \varphi \varphi \varphi$ & $9.660 \pm 0.395 * * * \mathrm{pDD} \delta \delta$ \\
\hline $7-24 \mathrm{~h}$ ON-OFF & $3.222 \pm 0.573 \varphi$ & $9.617 \pm 0.412 * * * \operatorname{pdp} \delta \delta$ \\
\hline
\end{tabular}

OT, ovarian torsion; h, hour; SR, simple reperfusion; g., gram; prot. protein. The results are presented as mean with standard deviation $\mathrm{M} \pm \mathrm{SD}$. Differences between groups compared to (and a $p$ value $<$ ):

$$
\begin{aligned}
& \text { - control: }{ }^{*}<0.05,{ }^{*} *<0.01,{ }^{*} * *<0.001 \text {; } \\
& \text { sham: }{ }^{\mathrm{D}}<0.05,{ }^{\mathrm{DD}}<0.01,{ }^{\mathrm{DDD}}<0.001 \text {; } \\
& \text { OT: } \bar{\delta}<0.05, \delta \delta<0.01, \delta \delta \delta<0.001 \text {; } \\
& \text { 1h SR: } \beta<0.05, \beta \beta<0.01, \beta \beta \beta<0.001 \text {; } \\
& \text { 1h ON-OFF: } \varphi<0.05, \varphi \varphi<0.01, \varphi \varphi \varphi<0.001 \text {; } \\
& \text { 24h SR: } \mathrm{s}<0.05, \mathrm{~s} s<0.01 \text {, } \mathrm{s} s \mathrm{~s}<0.001 \text {; } \\
& \text { 24h ON-OFF: } \int<0.05 \text {, } \int \mathrm{J}<0.01 \text {, } \int \mathrm{J}<0.001
\end{aligned}
$$


Table 2. Lactate and AOPP levels in blood serum

\begin{tabular}{|c|c|c|}
\hline Group & Lactate, $\mathrm{mmol} / \mathrm{L}$ & $\mathrm{AOPP}, \mu \mathrm{mol} / \mathrm{L}$ \\
\hline 1 - control & $3.014 \pm 0.301$ & $26.415 \pm 3.251$ \\
\hline 2 - sham & $3.071 \pm 0.388$ & $26.474 \pm 3.543$ \\
\hline 3 - ischemia (OT) & $\begin{array}{l}5.029 \pm 0.442 * * * \operatorname{DDD} \beta \beta \beta \\
\varphi \varphi \varphi \int\end{array}$ & $31.885 \pm 2.322 * \mathrm{D} \varphi \varphi \iint J$ \\
\hline $4-1 h$ SR & $6.632 \pm 0.284 * * * \operatorname{pDD} \delta \delta \delta$ & $33.412 \pm 3.454 * * \mathrm{DD} \varphi \varphi \iint$ \\
\hline 5 - 1h ON-OFF & $6.590 \pm 0.335 * * * \operatorname{pDD} \delta \delta \delta$ & $27.824 \pm 1.566 \delta \delta \beta \beta$ sss \\
\hline $6-24 h$ SR & $5.633 \pm 1.151 * * * \mathrm{pdp}$ & $33.234 \pm 1.892 * * \mathrm{DD} \varphi \varphi \varphi \iint J$ \\
\hline $7-24 \mathrm{~h}$ ON-OFF & $6.254 \pm 0.952 * * * \operatorname{pod} \delta$ & $26.580 \pm 1.800 \delta \delta \delta \beta \beta$ ss \\
\hline
\end{tabular}

OT, ovarian torsion; $h$, hour; SR, simple reperfusion; $L$, litre. The results are presented as mean with standard deviation - M \pm SD. Differences between groups compared to (and a $p$ value $<$ ):

$$
\begin{aligned}
& \text { control: *<0.05, }{ }^{* *}<0.01,{ }^{* * *} * 0.001 \text {; } \\
& \text { sham: }{ }^{\mathrm{D}}<0.05, \mathrm{DD}^{\mathrm{D}}<0.01, \mathrm{DDD}^{\mathrm{D}}<0.001 \\
& \text { OT: } \delta<0.05, \delta \delta<0.01, \delta \delta \delta<0.001 \text {; } \\
& \text { 1h SR: } \beta<0.05, \beta \beta<0.01, \beta \beta \beta<0.001 \text {; } \\
& \text { 1h ON-OFF: } \varphi<0.05, \varphi \varphi<0.01, \varphi \varphi \varphi<0.001 \text {; } \\
& \text { 24h SR: } \mathrm{s}<0.05, \mathrm{~s}<<0.01 \text {, } \mathrm{s} \mathrm{s}<0.001 \text {; } \\
& \text { 24h ON-OFF: } \int<0.05 \text {, IJ }<0.01 \text {, IJ }<0.001 \text {. }
\end{aligned}
$$

Ischemic processes determined the orientation of metabolism to lactate production. Its serum and ovarian homogenates values in the control and sham group were not statistically significantly different, $p>0.05$. After ovarian ischemia, the amount of lactate increased in serum by about $66 \%$ (from $3.014 \pm 0.301 \mathrm{mmol} / \mathrm{L}$ to $5.029 \pm 0.442 \mathrm{mmol} / \mathrm{L}, \mathrm{p}<0.001$ ) and in homogenates by about $38 \%$ (from $2.893 \pm 0.283 \mathrm{mmol} / \mathrm{g}$.prot. to $4.002 \pm 0.677 \mathrm{mmol} / \mathrm{g}$.prot., $\mathrm{p}=0.006$ ) in the OT group, compared to the control group.

The serum lactate (Table 2), after 1 hour, in both simple and controlled reperfusion groups, had a statistically significant growth by about $120 \%$ compared to control group (from $3.014 \pm 0.301$ $\mathrm{mmol} / \mathrm{L}$ to $6.632 \pm 0.284 \mathrm{mmol} / \mathrm{L}$ in 1 hour $\mathrm{SR}$, respectively to $6.590 \pm 0.335 \mathrm{mmol} / \mathrm{L}$ in 1 hour ON-OFF, $\mathrm{p}<0.001)$. Compared to the group of rats exposed to ovarian ischemia only (the $3^{\text {rd }}$ group), the increase of serum lactate was about $31 \%$ (from $5.029 \pm 0.442$ $\mathrm{mmol} / \mathrm{L}$ to $6.632 \pm 0.284 \mathrm{mmol} / \mathrm{L}$ in 1 hour $\mathrm{SR}$, respectively to $6.590 \pm 0.335 \mathrm{mmol} / \mathrm{L}$ in 1 hour ON-OFF, $\mathrm{p}<0.001$ ). Subsequently, at 24 hours after the restoration of the blood circulation, no significant decrease was registered compared to 1 hour groups, $p>0.05$. However, there was established a tendency to lower the initial values recorded after 1 hour of reperfusion, this being more evident in the 24 hours simple reperfusion than in 24 hours controlled reperfusion.

Removal of ischemia, a phenomenon involving the restoration of the blood flow with significant oxygen and nutrient intake, no longer determines the increase in lactate levels in the ovaries (Table 1). The amounts recorded in 1 hour SR group and 1 hour ON-OFF group were not significantly different from those recorded in the OT group, $p>0.05$. The trend of this index in the homogenates is to return to baseline after 24 hours of reperfusion in both groups with simple and controlled reperfusion.

There were no correlations between the amounts of lactate determined in the blood serum and ovarian homogenates in the research groups, except the moderate positive correlation in the sham group $\left(r_{s}=0.673, p=0.033\right)$.

Ovarian torsion induced an increase in AOPP. Its serum and ovarian homogenates values in the control and sham group were not different, $p>0.05$. Ischemia determined a significant growth of AOPP in serum by about $20 \%$ compared to control (from $26.415 \pm 3.251 \mu \mathrm{mol} / \mathrm{L}$ to $31.885 \pm 2.322 \mu \mathrm{mol} / \mathrm{L}, \mathrm{p}=0.007)$. AOPP increased non-singnificantly after 1 hour and 24 hours of simple reperfusion compared to OT group ( $p>0.05)$, while in the ON-OFF groups AOPP values decreased and were lower compared to those recorded in SR groups by about $20 \%$ (from $33.412 \pm 3.454 \mu \mathrm{mol} / \mathrm{L}$ in $1 \mathrm{~h}$ SR group to $27.824 \pm 1.566 \mu \mathrm{mol} / \mathrm{L}$ in $1 \mathrm{~h}$ ON-OFF group, $\mathrm{p}=0.007$, respectively $26.580 \pm 1.800 \mu \mathrm{mol} / \mathrm{L}$ in $24 \mathrm{~h}$ ON-OFF group, $p=0.001$, and from $33.234 \pm 1.892 \mu \mathrm{mol} / \mathrm{L}$ in $24 \mathrm{~h}$ SR group, to $27.824 \pm 1.566$ $\mu \mathrm{mol} / \mathrm{L}$ in $1 \mathrm{~h}$ ON-OFF group, $\mathrm{p}<0.001$, respectively $26.580 \pm 1.800$ $\mu \mathrm{mol} / \mathrm{L}$ in $24 \mathrm{~h}$ ON-OFF group, $\mathrm{p}<0.001$ ).

Ovarian torsion determined an increased of AOPP content in the ovaries by about $26 \%$ (from $5.695 \pm 0.781 \mu \mathrm{mol} / \mathrm{g}$.prot. to $7.183 \pm 1.566 \mu \mathrm{mol} / \mathrm{g}$.prot., $\mathrm{p}>0.05$ ) compared to control group. AOPP levels rised after reperfusion despite its technique. At 1 hour of detorsion the AOPP was higher in SR group compared to ischemia group $(p=0.016)$. The amounts registered in 1 hour ONOFF group were not significantly different from those recorded in OT group ( $p>0.05$ ). At 24 hours of reperfusion the values of AOPP rised by about $34 \%$ compared to ischemia group (from $7.183 \pm 1.566 \mu \mathrm{mol} / \mathrm{g}$.prot. in OT group to $9.660 \pm 0.395$ $\mu \mathrm{mol} / \mathrm{g}$.prot. in $24 \mathrm{~h}$ SR group, $\mathrm{p}=0.008$, respectively to $9.617 \pm 0.412 \mu \mathrm{mol} / \mathrm{g}$.prot. in $24 \mathrm{~h}$ ON-OFF group, $\mathrm{p}=0.009$ ).

No correlations were found between the levels of AOPP from the blood serum and ovarian homogenates in the research groups, except the strong negative correlation in the 24 hours ON-OFF group $\left(r_{s}=-0.770, p=0.009\right)$.

\section{Discussion}

Ovarian torsion is accompanied by oxidative stress, AOPP and lactate synthesis.

It is established that the disease is followed by ischemiareperfusion injuries due to an enhance of oxidative stress [2]. Different enzymes are responsible for this process, one of them being myeloperoxidase (MPO) from neutrophils. MPO is the main enzyme involved in the formation of AOPP. ROS that are produced by this MPO have an important role in changing the structure of proteins to dityrosine containing and cross-linked structures. Hypochlorous acid $(\mathrm{HClO})$ and chloramine $\left(\mathrm{NH}_{2} \mathrm{Cl}\right)$ directly transform proteins into AOPP [13].

Analyzing our results, the blood serum low level of AOPP in the ON-OFF reperfusion groups compared to SR groups indicates a decrease in ROS production when controlled method of reperfusion is used.

As AOPP are known to be compounds with negative effect that increase the inflammatory response [13], the lower serum values in controlled reperfusion groups compared to SR groups suggest that ON-OFF method could prevent other possible lesions in the organ and at distance from the injured ovary. Thus, one of the suppositions of our research results is that the controlled type of reperfusion in ovarian torsion could have protective effect on other organs and tissues, reducing the ROS production and consequently inflammatory reactions due to AOPP.

Less obvious increase of AOPP in ovarian homogenates during the initial period of controlled reperfusion (at 1 hour after the restoration of the blood flow), followed by a significant reduction of differences between groups at distance (after 24 hours), suggests the protective effect of ON-OFF technique in the early period.

Piwowar A. (2010) mentioned that AOPP interact with specific RAGE receptors and stimulates the activity of neutrophils and the 
inflammatory response. Enzymes such as NADPH oxidase and MPO also increase their activity. Thus, ROS production is increased and as a consequence the AOPP too [13]. It is well known that RAGE are expressed in ovarian cells [15]. Then, the high levels of AOPP identified in ovarian homogenates at 24 hours of reperfusion can be due to an increased inflammatory response, as a consequence of interaction of AOPP that were produced in the initial period of reperfusion with RAGE. Thereby is supported the hypothesis of a vicious circle where AOPP growth stimulates the production of other AOPP in tissues, which are subsequently identified at 24 hours of reperfusion in our research.

As 1 hour after reperfusion, lower amounts of AOPP were determined in homogenates of controlled reperfusion group than in SR group, it can be concluded that the ON-OFF procedure could have protective effect at early stages of treatment. However, the lack of differences between AOPP values in both types of reperfusion after 24 hours indicates the need for antioxidants to be used as soon as possible after diagnosis to prevent the enhancement of lesions.

Under reduced tissue perfusion, anaerobic processes, as anaerobic glycolysis, become intense, thus, increasing the lactate. The increase in lactate level in the ovarian homogenate is the result of preferential anaerobic oxidation of glucose. The approximately two-fold increase in the amount of serum lactate in the reperfusion groups compared to control group, indicates a takeover of lactate at the time of reperfusion from the tissue.

The high metabolism of leukocytes infiltrating the organ after ischemia could also increase lactate. A high level of AOPP in the homogenates after the restoration of blood flow indicates an important activity of these cells, AOPP being synthesized under the action of MPO that belong to leukocytes, these growing lactate too. This hypothesis requires further research.

After 24 hours of reperfusion, the lactate level tendend to decrease in the serum. This could be due to liver that metabolizes lactate from peripheral tissues during the Cori cycle [16] or to other organs that take up and oxidize it [12].

The change in lactate transporters, these transporters being membrane proteins [16], could reduce the lactate clearance rate. Thus, high levels of lactate identified in the homogenates of groups exposed to ovarian torsion, could be due also to affected membrane proteins by oxidative stress.

We must cautiously interpret the changes of these parameters in the blood serum because there were not found homogenateserum correlations of the studied indices in all research groups. A decreased in AOPP in serum did not show a similar trend of decreasing in homogenates. Thereby, organ-level changes can not be appreciated based on the blood level of AOPP or lactate.

\section{Conclusion}

Our results suggest that lactic acid is a biomarker for ischemia in ovarian torsion, reflecting the anaerobic changes of metabolic processes and also the high metabolism of neutrophils infiltrating the organ after ischemia.

The increased AOPP in experimental groups indicate that there is a degree of oxidative stress that can affect the cells after the restoration of the blood flow and then the use of antioxidants could be helpful in the early stages of the disease. As AOPP were higher in simple reperfusion groups compared to ON-OFF groups, the controlled reperfusion method in ovarian torsion could reduce reactive oxygen species production and could be appropriate to prevent additional injuries due to reperfusion.

\section{Limitations}

This study has some limitations. First, the experimental induced ovarian torsion is not identical to what is encountered in clinical practice. But, to conduct such a research in humans is difficult because the disease is rare, clinical signs are not specific and often mislead doctors. Second, it is well known that there are interspecies differences concerning different parts of metabolism, then, the research results can not be directly related to humans. Further studies are required to establish if these experimental results are applicable in humans.

\section{Funding}

Doctoral grant offered by Ministry of Education, Culture and Research of Republic of Moldova.

\section{Conflict of interest}

No conflicts of interest.

\section{Ethical approval}

The study protocol was approved by the Research Ethics Committee of the Nicolae Testemitanu State University of Medicine and Pharmacy (08.02.2016). All applicable international, national, and/or institutional guidelines for the care and use of animals were followed. All procedures performed in studies involving animals were in accordance with the ethical standards of the institution or practice at which the studies were conducted.

\section{References}

1. Zucchini S, Marra E. Diagnosis of emergencies/urgencies in gynecology and during the first trimester of pregnancy. J Ultrasound 2014; 17(1): 41-46. https://doi.org/10.1007/s40477-013-0059-0.

2. Tuncer AA, Bozkurt MF, Koken T, Dogan N, Pektaş MK, Baskin Embleton D. The Protective Effects of Alpha-Lipoic Acid and Coenzyme Q10 Combination on Ovarian Ischemia-Reperfusion Injury: An Experimental Study. Adv Med 2016; 2016: 3415046. http://doi.org/10.1155/2016/3415046.

3. Vijayalakshmi K, Reddy GMM, Subbiah VN, Sathiya S, Arjun B. Clinicopathological profile of adnexal torsion cases: a retrospective analysis from a tertiary care teaching hospital. J Clin Diagn Res 2014; 8(6): OC04-OC07. https://doi.org/10.7860/JCDR/2014/8167.4456.

4. Bolli P, Schädelin S, Holland-Cunz S, Zimmermann P. Ovarian torsion in children: Development of a predictive score. Medicine (Baltimore) 2017; 96(43): e8299. https://doi.org/10.1097/MD.0000000000008299.

5. Yildirim A, Yildirim S, Topaloglu N, Tekin M, Kucuk A, Erdem H, et al. Correlation of ischemia-modified albumin levels and histopathologic findings in experimental ovarian torsion. Turk J Emerg Med 2016; 16(1): 8-11. https://doi.org/10.1016/j.tjem.2016.02.004.

6. Nair S, Joy S, Nayar J. Five year retrospective case series of adnexal torsion. J Clin Diagn Res 2014; 8(12): OC09-OC13. https://doi.org/10.7860/JCDR/2014/9464.5251.

7. Pages-Bouic E, Millet I, Curros-Doyon F, Faget C, Fontaine M, Taourel $P$. Acute pelvic pain in females in septic and aseptic contexts. Diagn Interv Imaging 2015; 96(10): 985-995. https://doi.org/10.1016/i.diii.2015.07.003.

8. Poonai N, Poonai C, Lim R, Lynch T. Pediatric ovarian torsion: case series and review of the literature. Can J Surg 2013; 56(2): 103-108. https://doi.org/10.1503/cjs.013311.

9. Ercan Ö, Köstü B, Bakacak M, Coşkun B, Tohma A, Mavigök E. Neutrophil to Lymphocyte ratio in the diagnosis of adnexal torsion. Int 
$J \quad$ Clin Exp Med 2015; 8(9): 16095-16100. https://www.ncbi.nlm.nih.gov/pubmed/26629118.

10. Karatas Gurgun A, Kaban I, Aka N, Mentese A, Aker F, Uras AR. The Role of ischemia Modified Albumin and D-dimer as Early or Late Biochemical Markers in Ovarian Torsion. J Obstet Gynaecol Res 2017; 43(5): 895-901. https://doi.org/10.1111/iog.13281.

11. Laganà AS, Sofo V, Salmeri FM, Palmara VI, Triolo O, Terzić MM, et al. Oxidative stress during ovarian torsion in pediatric and adolescent patients: changing the perspective of the disease. Int J Fertil Steril 2016; 9(4): 416-423. https://doi.org/10.22074/ijfs.2015.4598.

12. Gladden LB. Lactate metabolism: a new paradigm for the third millennium. J Physiol 2004; 558(Pt 1): 5-30. https://doi.org/10.1113/iphysiol.2003.058701.

13. Piwowar A. Advanced oxidation protein products. Part I. Mechanism of the formation, characteristics and property. Pol Merkur Lekarski 2010; 28(164): 166-169. Polish. https://www.ncbi.nlm.nih.gov/pubmed/20369749.

14. Capeillère-Blandin C, Gausson V, Descamps-Latscha B, Witko-Sarsat V. Biochemical and spectrophotometric significance of advanced oxidized protein products. Biochim Biophys Acta 2004; 1689(2): 91-102. https://doi.org/10.1016/j.bbadis.2004.02.008.

15. Stensen MH, Tanbo T, Storeng R, Fedorcsak P. Advanced glycation end products and their receptor contribute to ovarian ageing. Hum Reprod 2014; 29(1): 125-134. https://doi.org/10.1093/humrep/det419.

16. Sun S, Li H, Chen J, Qian Q. Lactic acid: no longer an inert and endproduct of glycolysis. Physiology (Bethesda) 2017; 32(6), 453-463. https://doi.org/10.1152/physiol.00016.2017.

Authors:

Cornelia Lazăr - PhD fellow, assistant, Chair of Biochemistry and Clinical Biochemistry, Nicolae Testemitanu State University of Medicine and Pharmacy, Chisinau, Republic of Moldova. https://orcid.org/0000-00018056-9608.

Marin Vozian - MD, PhD, First Department of Surgery "Nicolae Anestiadi", Nicolae Testemitanu State University of Medicine and Pharmacy, Chisinau, Republic of Moldova, https://orcid.org/0000-0001-9760-2056.

Valeriana Pantea - scientific researcher, Laboratory of Biochemistry, Nicolae Testemitanu State University of Medicine and Pharmacy, Chisinau, Republic of Moldova. https://orcid.org/0000-0002-8835-6612.

Inna Svet - scientific researcher, Laboratory of Biochemistry, Nicolae Testemitanu State University of Medicine and Pharmacy, Chisinau, Republic of Moldova. https://orcid.org/0000-0001-6059-1170.

Anna Mishina - MD, PhD, Head of Surgical Gynecology Department, Mother and Child Institute, Chisinau, Republic of Moldova. https://orcid.org/0000-0002-8317-422X.

Olga Tagadiuc - MD, PhD, Head of Biochemistry and Clinical Biochemistry Chair of Nicolae Testemitanu State University of Medicine and Pharmacy, Chisinau, Republic of Moldova. https://orcid.org/0000-0002-5503-8052. 